\title{
Apropiación social de tecnologías digitales por jóvenes universitarios mayas de Quintana Roo
}

\section{Social appropriation of digital technologies by young Maya students of higher education from Quintana Roo}

\section{Apropriação social de tecnologias digitais por jovens universitários maias} de Quintana Roo

\section{Dulce Angélica Gómez Navarro}

Centro de Investigaciones y Estudios Superiores en Antropología Social (CIESAS), México

dgomeznavarro@ciesas.edu.mx https://orcid.org/0000-0003-4571-3250

\section{Resumen}

La brecha digital es un problema que se articula con otras desigualdades y afecta a poblaciones en condiciones de pobreza. Así, la mayoría de las regiones indígenas en México presentan dificultades para conectarse, a pesar de que el uso del teléfono celular ha proliferado en la última década en zonas rurales, donde los jóvenes son los usuarios más activos. Este estudio etnográfico retoma el concepto apropiación social de las tecnologías de la información y la comunicación (TIC) desde una perspectiva sociocultural para comprender su aprovechamiento y para analizar los factores sociales que inciden en la apropiación tecnológica de jóvenes mayas de la Universidad Intercultural Maya de Quintana Roo (UIMQROO), los cuales, a pesar de que cuentan con experiencias escolares discontinuas en la adquisición de habilidades digitales, usan cotidianamente las TIC y las redes sociales como parte de la necesidad de pertenecer a la era digital.

Palabras clave: brecha digital, educación superior, iniciación a la informática, población indígena, tecnologías de la información. 


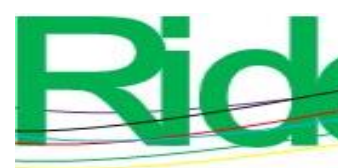
Revista Iberoamericana para la
Investigación y el Desarrollo Educativo
ISSN $2007-7467$

\section{Abstract}

The digital divide is an inequality that is linked to other inequalities and affects populations living in poverty. Thus, most indigenous regions in Mexico have difficulties to connect, however, cell phone use has proliferated in rural areas in the last decade and young people are the most active users. This ethnographic study takes up the concept of social appropriation of Information and Communication Technologies (ICT) from a sociocultural perspective to understand the use of digital technologies and analyzes the social factors that influence the technological appropriation of young Mayans from the University Intercultural Maya de Quintana Roo (UIMQROO), which, despite having limited and intermittent school experiences in the acquisition and use of ICT, daily use ICT and social networks as part of the need to belong to the digital age.

Keywords: digital divide, higher education, digital literacy, indigenous people, ICT.

\section{Resumo}

A exclusão digital é um problema que está ligado a outras desigualdades e afeta as populações que vivem na pobreza. Assim, a maioria das regiões indígenas do México tem dificuldade de se conectar, embora o uso do telefone celular tenha proliferado na última década nas áreas rurais, onde os jovens são os usuários mais ativos. Este estudo etnográfico retoma o conceito de apropriação social das tecnologias de informação e comunicação (TIC) numa perspectiva sociocultural para compreender a sua utilização e analisar os fatores sociais que influenciam a apropriação tecnológica de jovens maias da Universidade Intercultural Maya de Quintana Roo (UIMQROO ), que apesar de terem experiências escolares descontínuas na aquisição de competências digitais, utilizam as TIC e as redes sociais no quotidiano como parte da necessidade de pertencer à era digital.

Palavras-chave: exclusão digital, ensino superior, iniciação à computação, população indígena, tecnologia da informação.

Fecha Recepción: Abril 2021

Fecha Aceptación: Octubre 2021 


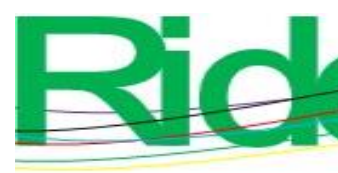

Revista Iberoamericana para la
Investigación y el Desarrollo Educativo
ISSN $2007-7467$

El documento tiene la siguiente estructura: en el subapartado que compone la introducción se analiza la desigualdad digital y los pueblos indígenas en México, se revisa la definición de apropiación social de TIC y se sitúa a los jóvenes indígenas en su integración a la era digital. Posteriormente, se enuncia la metodología. Los resultados se presentan con base en los hallazgos más significativos del estudio: brecha digital y usos de las tecnologías digitales, trayectorias educativas y alfabetización digital, y usos significativos de las TIC y las redes sociales. Por último, se exponen la discusión y las conclusiones.

\section{Desigualdad digital y los pueblos indígenas en México}

Son pocos los estudios que se han realizado en torno a caracterizar la brecha digital que afecta a las comunidades indígenas en el mundo (Borrero, 2016) y en México. Algunos de los estudios sobre los pueblos indígenas y la era digital son los de Ramos Mancilla (2020), Soto-Hernández, Valencia-López y Rentería-Gaeta (2020) y Bonilla, Cruz y Pérez (2018). En cuanto a investigaciones de uso de TIC en la educación superior indígena, Tipa (2019) y Ortiz y Alarcón (2014) evidenciaron las distintas desigualdades que presentan los estudiantes debido a una escolaridad previa deficiente en materia tecnológica. Otro estudio muestra la brecha digital en la que se encuentran los jóvenes del Instituto Superior Ayuuk (ISIA) y la poca alfabetización tecnológica que realizan los profesores (Vergara, 2015).

Tipa (2019) refiere la relación entre el estrato socioeconómico y el uso de las TIC en la Universidad Intercultural de Chiapas (UNICH), donde los estudiantes de nivel económico bajo presentan mayores dificultades para poseer laptops y memorias USB. Asimismo, se encontraron diferencias etnolingüísticas debido a que los alumnos que hablan una lengua materna cuentan con menos dispositivos electrónicos y se conectan menos a internet y a las redes sociales.

Como parte de las desigualdades sociales, el acceso a las TIC no es posible para todas las personas. Desde 1995 — cuando surgió el concepto brecha digital (van Dijk, 2017) — se pueden identificar tres periodos de investigación: el primero trata sobre el acceso físico a la tecnología. El segundo evidencia que no es suficiente con tener acceso, sino que depende del empleo significativo de las TIC. El tercer periodo integra los resultados que tiene el uso de las TIC y los beneficios individuales y colectivos, donde se muestra que las personas en entornos más privilegiados tienen una mayor participación digital (Robinson et al., 2020). 


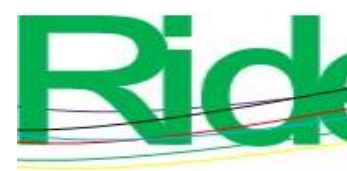

Revista Iberoamericana para la
Investigación y el Desarrollo Educativo
ISSN $2007-7467$

Se estima que en los países con mayores niveles de pobreza la adquisición de un dispositivo tecnológico y la conexión a internet son más difíciles que en los países desarrollados. Así, la brecha digital mundial se caracteriza por afectar más a zonas rurales, indígenas y pobres, a mujeres, a personas pobres y con baja escolaridad y a adultos mayores (Borrero, 2016).

La desigualdad en el acceso y uso de las TIC está asociada a distintos factores económicos, geográficos, sociales, culturales, lingüísticos, de género, educativos y generacionales (Robinson et al., 2020). Algunas investigaciones destacan la relación de pobreza y desigualdad digital, donde las familias con mayor capacidad económica tienen más acceso a las TIC (Sunkel, 2006).

En México, la Encuesta Nacional sobre Disponibilidad y Uso de Tecnologías de la Información en los Hogares (Endutih) es el mecanismo para obtener datos respecto al acceso y uso de las TIC; no obstante, se centra en localidades urbanas e incluye un bajo porcentaje de población rural en localidades menores de 2500 habitantes (Inegi, 2020a).

Es relevante señalar que ni la población indígena ni afrodescendiente se incluyen en la encuesta, por lo que existe un vacío importante. En esta línea, en un estudio de 2018 sobre uso tecnológico y pueblos indígenas, realizado por el Instituto Federal de Telecomunicaciones (IFT), se concluye que este sector utiliza un servicio de baja velocidad (2G y 3G) (Instituto Federal de Telecomunicaciones [IFT], 2018).

Por lo que respecta a la diferencia entre las zonas rurales y las urbanas, se encontró que mientras la conexión a internet de las personas urbanas oscila entre $76.6 \%$, en las zonas rurales baja a $47.7 \%$. Lo mismo sucede con la disponibilidad de computadora, donde 50.9 $\%$ de los habitantes rurales tienen una, y solo $20.6 \%$ en el ámbito rural (Inegi, 2020a).

Las disparidades que se observan en los datos provienen principalmente de carencias en infraestructura tecnológica, la marginación, la pobreza (Inegi, 2020b) y los bajos niveles de escolaridad (Inegi, 2020a).

Por ejemplo, mientras que la población con educación superior a nivel nacional se conecta a internet en $96.4 \%$, quienes solo tienen educación primaria lo hacen en $59.1 \%$ (Inegi, 2020a). Las principales causas por las que los habitantes no poseen TIC son económicas (Inegi, 2020b). 


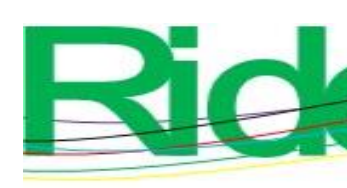

Revista Iberoamericana para la Investigación y el Desarrollo Educativo ISSN $2007-7467$

Finalmente, la población con mayor brecha digital son las personas mayores de 55 años, lo que se agrava en mayor medida en el sector rural, donde solo $3.8 \%$ de los adultos en ese rango de edad utilizan la tecnología (Inegi, 2020b).

Respecto a las políticas que se han puesto en marcha para garantizar el derecho constitucional al uso de las TIC, destacan diversos programas derivados de la Estrategia Digital Nacional, como México Conectado o Agenda Digital Mx para fortalecer la infraestructura y dotar de habilidades digitales a la población. Desafortunadamente son pocos los avances en el cierre de la brecha digital rural e indígena (Soto-Hernández et al., 2020) porque han sido proyectos enfocados a la población urbana principalmente. Lo anterior evidencia la poca inversión y los altos niveles de exclusión digital que tiene el país (Mariscal, 2020), especialmente en el campo de la educación (Instituto Nacional para la Evaluación de la Educación [INEE], 2019).

En el caso de la población indígena en México, el rezago educativo es mayor frente a la población no indígena por distintas carencias en cuanto a cobertura, equipamiento, infraestructura, calidad de la educación, capacitación, entre otros. Esto a su vez se relaciona con la brecha digital rural, la deficiente educación y los altos niveles de pobreza (Secretaría de Comunicaciones y Transportes [SCT], 2019).

Un ejemplo es la evidente inequidad en la distribución de equipos de cómputo según datos del INEE (2019), así como "la ausencia de políticas o lineamientos normativos federales o estatales para la distribución de computadoras en los distintos tipos de servicio" (p. 233). El sistema educativo presenta un rezago tecnológico muy importante, donde solo en el ciclo 2017-2018, 46.5\% de las escuelas primarias contaban con al menos una computadora y $31.9 \%$ de las escuelas indígenas tenían este servicio. En el nivel secundaria se contabiliza $74.9 \%$ a nivel nacional. En lo que respecta a conexión a internet, $48.1 \%$ de las primarias públicas tienen conectividad, frente a $13.4 \%$ de las primarias indígenas y 5.5 $\%$ de las primarias comunitarias (INEE, 2019). Asimismo, sobresalen las pocas habilidades digitales que poseen las plantillas docentes.

Así, el rezago tecnológico de la población indígena se debe a que "esta desigualdad se origina por condiciones de vulnerabilidad económica, social y cultural" (Bonilla et al., 2018, p. 72); por esta razón, los proyectos comunitarios de uso tecnológico avanzan lento y con limitaciones, como lo refieren estos autores respecto a la creación de contenidos en lenguas indígenas en el ciberespacio. 


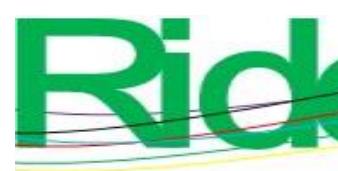

Revista Iberoamericana para la
Investigación y el Desarrollo Educativo
ISSN $2007-7467$

internet y $87.8 \%$ declara ser usuario de redes sociales (Facebook, WhatsApp, Twitter e Instagram son las más empleadas) (Inegi, 2020a).

Así, la tradición y la modernidad se entrecruzan y los consumos culturales se adaptan a los nuevos tiempos. En el caso de los jóvenes indígenas, aprovechan esta deslocalización inherente a la globalización, y se apropian de las TIC y reconfiguran identidades (Pérez Ruiz, 2019) con un fuerte componente de sentido de pertenencia al movimiento global y utilizan a las TIC como emblemas de esa cultura digital en función de sus condiciones contextuales y sus activos sociales.

Las experiencias de socialización presenciales se amplían con el mundo virtual (Lévy, 2007), al mismo tiempo que "son reproductoras y generadoras de una realidad online paralela a la realidad offline" (Gallego, 2016, p. 33). Por un lado, las plataformas virtuales fungen como un medio de representación y exhibición de imaginarios sociales y, por otro, configuran y proponen formas de agrupamiento y de identificación social que incluyen pautas de comportamiento, prácticas, usos y patrones de consumo (León-Pasquel, 2018).

En cuanto a la participación de estos jóvenes en la era digital, ha emergido un nuevo papel como mediadores digitales (Hepp, 2009). Para Pérez Ruiz (2019) invierten su posición de subordinación que la estructura social les ha impuesto debido a que por primera vez son ellos quienes presentan mayores habilidades digitales para enseñar a las personas mayores, y no al revés, como históricamente ha sucedido en otros ámbitos.

Para Bonilla et al. (2018), "los jóvenes reinventan la tecnología por medio del trabajo colaborativo, el talento creativo y por medio de alianzas estratégicas para consolidar una sofisticada combinación del lenguaje informático y del código cultural” (p. 72). Estas prácticas demuestran cómo es posible la apropiación tecnológica en los pueblos originarios. La apropiación social de las TIC se refiere al proceso en que las personas otorgan un uso benéfico a ellas ya sea a nivel individual o social, en función de las condiciones socioculturales, económicas, políticas, tecnológicas, educativas demográficas y geográficas (Andrés, 2014).

Desde una perspectiva sociocultural de la tecnología, la cultura es una dimensión constitutiva y constituyente en el uso de las TIC (Meneses, 2019) debido a que los artefactos tienen un significado particular (Lévy, 2007; Santos, 2003). De esta forma, las TIC inciden en la vida cotidiana y configuran prácticas sociales (Winocur, 2015). 


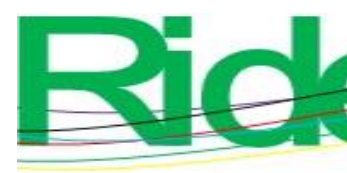

Revista Iberoamericana para la
Investigación y el Desarrollo Educativo
ISSN $2007-7467$

De acuerdo con Crovi Druetta y López-González (2011), la apropiación social “se concreta en un ámbito sociohistórico específico en el cual el individuo no sólo tiene acceso a ellas, sino que cuenta con habilidades para usarlas y llegan a ser tan importantes para sus actividades cotidianas (productivas, de ocio, relacionales) que pasan a formar parte de sus prácticas sociales" (p. 74).

La habilidad digital se concibe como el nivel de dominio que posee el individuo para manejar las TIC, la cual requiere capacitación técnica específica. Para van Dijk (2017) y Cobo et al. (2018), la apropiación de las tecnologías está mediada por un acceso y una motivación en el uso, así como una alfabetización digital. Otros determinantes son la escolaridad, la posición socioeconómica, el género, el equipamiento tecnológico, el origen étnico y la edad, por mencionar algunas.

Para Lévy (2007) en el ciberespacio la inteligencia personal que precede a la inteligencia colectiva requiere de la adquisición primaria de habilidades digitales básicas, pero también de un nuevo alfabetismo que dota de la capacidad de clasificar e interpretar la información en una virtualidad que se desborda por su crecimiento exponencial y que incluye desinformación. Así, la selección de datos se vuelve imprescindible. La inteligencia colectiva es la función central de la era digital que el autor identifica debido a las posibilidades que alberga el ciberespacio como un espacio abierto, socializante y participativo.

Asimismo, las trayectorias educativas, las motivaciones en el uso y los activos económicos y sociales son aspectos que se entretejen en las biografías de los jóvenes indígenas para acceder, usar y apropiarse de las TIC, que se verán a continuación. Es decir, la escolaridad es un factor clave porque permite obtener habilidades digitales, pero también las condiciones comunitarias tanto de infraestructura y equipamiento como de redes familiares y de amistad, que inciden en el uso o no uso y motivan el aprendizaje tecnológico.

\section{Metodología}

La investigación se realizó en la Universidad Intercultural Maya de Quintana Roo (UIMQROO), ubicada en localidad de José María Morelos (JMM), que se encuentra en el sur del estado. Esta institución alberga a más de 700 estudiantes (principalmente mayas) que 


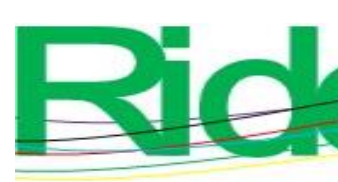

Revista Iberoamericana para la Investigación y el Desarrollo Educativo ISSN $2007-7467$

estudian alguno de los 10 programas educativos ${ }^{1}$ de más de 60 comunidades indígenas y rurales aledañas a la cabecera municipal de José María Morelos, tanto de Quintana Roo como de Yucatán.

Esta investigación etnográfica (Guber, 2011; Velasco y Díaz de Rada, 1997) integró una revisión bibliográfica extensa sobre las temáticas que aborda el objeto de estudio para la construcción del estado del arte, una reflexión conceptual para elaborar el marco teórico que retoma un abordaje sociocultural y antropológico de la tecnología, un análisis de las condiciones sociodemográficas y económicas del contexto del estudio y una etapa de trabajo de campo donde se recolectó la información empírica, tomando como base aspectos clave propuestos por la antropología de la educación.

Es relevante señalar que el valor del trabajo etnográfico en el campo de la educación consiste en reconocer lo genérico que se expresa en cada caso y describir una realidad educativa y social mediante el trabajo de campo, entendido como un "lugar para vivir" (Velasco y Díaz de Rada, 1997, p. 105). Este método cualitativo permite comprender los significados del contexto cultural en donde se inserta el fenómeno, tratando de desentrañar las concepciones de los actores sociales que corresponde a la perspectiva emic, para posteriormente cruzarla con la perspectiva etic, más de carácter estructural y teórico.

El trabajo de campo se dividió en dos partes: en la primera, se realizó una estancia corta con el propósito de gestionar el acceso al campo e iniciar el proceso de construcción de confianza requerido. Esta visita sirvió para conocer la localidad de José María Morelos, la universidad y el personal académico para presentar la investigación y recibir retroalimentación respecto a los objetivos y el diseño de los instrumentos de recolección de datos. En la segunda estancia, que tuvo una duración de dos meses y medio en el año de 2017, se aplicaron las entrevistas y se llevaron a cabo los grupos focales. Cabe mencionar que la convivencia diaria se vuelve un elemento crucial que permite comprender y desentrañar "las reglas de acción social” (Velasco y Díaz de Rada, 1997, p. 91) y los significados del fenómeno que otorgan los actores en su contexto cultural.

El estudio conjuntó la realización de 8 entrevistas en profundidad a profesores y 10 a jóvenes universitarios (estudiantes y egresados), así como la ejecución de 6 grupos focales

\footnotetext{
${ }^{1}$ Licenciaturas en Salud Comunitaria, Lengua y Cultura, Turismo Alternativo, Gestión Municipal y Desarrollo y Gestión de las Artes. Ingenierías en Sistemas de Producción Agroecológicos, Desarrollo Empresarial y Tecnologías de la Información y Comunicación. Maestría en Educación Intercultural.
} 


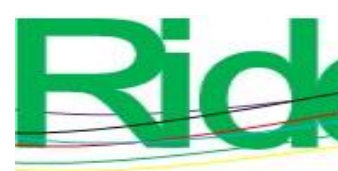

Revista Iberoamericana para la
Investigación y el Desarrollo Educativo
ISSN $2007-7467$

con la colaboración de 37 estudiantes en total, y la participación en varios eventos académicos de la universidad como gesto de reciprocidad en el proceso de investigación, en los que destacan clases magistrales y la elaboración de un instrumento de evaluación de habilidades digitales para estudiantes de primer semestre en colaboración con el cuerpo académico de la ingeniería en TIC . En todos los casos, se trabajó bajo consentimiento informado para respetar la confidencialidad de los participantes. Un elemento adicional fue la elaboración del diario de campo que acumuló más de 100 páginas de descripción etnográfica y observación participante.

La determinación de la muestra fue de acuerdo con un estudio de corte cualitativo e intencionado, es decir, se plantearon los actores clave de la investigación, misma que fue ajustada conforme a la primera visita. De esta forma, se buscó la representatividad y disponibilidad de estudiantes y egresados de diversas carreras de la universidad, así como de docentes y académicos. Algunas características de los estudiantes y los egresados es que sus edades oscilaron entre los 18 y 32 años, en su mayoría pertenecían a comunidades indígenas del sur de Quintana Roo y Yucatán, y la mayoría de los participantes hablaban el maya como primera lengua. En cuanto al personal académico, en esencialmente se entrevistó a profesores de asignatura de varias carreras y a la directora académica, quienes procedían prioritariamente de la región.

Posteriormente, las entrevistas y grupos focales fueron transcritos, los cuales se integraron a un corpus documental del que se realizó una primera codificación por medio del análisis de contenido (Bardin, 1996) y se utilizó el programa Atlas ti. En un segundo momento, se depuraron las categorías y subcategorías empíricas y se redactaron documentos de análisis (Restrepo, 2016). Finalmente, se realizó la "triangulación metodológica” (Taylor y Bodgan, 1987), que consiste en relacionar las principales categorías empíricas y sus múltiples asociaciones con las teorías y conceptos de la investigación

A continuación, se presentan los resultados de tres aspectos centrales que se obtuvieron de este proceso analítico y que dan cuenta de los factores que inciden en la apropiación tecnológica de los jóvenes universitarios: los usos de las diversas tecnologías digitales en un contexto de brecha digital, las trayectorias educativas y los procesos de alfabetización digital, y por último los significados del uso de las TIC y las redes sociales. 


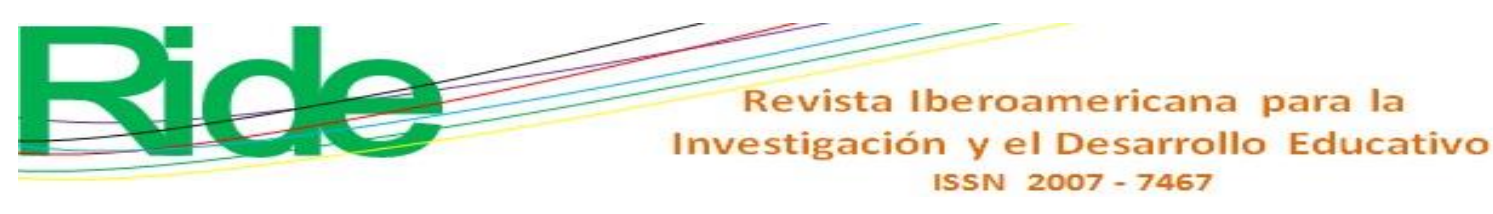

Resultados

\section{La brecha digital y los usos de las TIC}

José María Morelos (JMM) se caracteriza por ser un municipio con alto nivel de marginación y pobreza; tiene alrededor de 39165 habitantes, de los cuales $49.80 \%$ habla lengua indígena (maya con $99.6 \%$ ). Además, $2.17 \%$ de los habitantes se consideran afrodescendientes (Inegi, 2021, p. 21). Las principales actividades económicas son la agricultura y la silvicultura, seguidas del comercio. En los últimos 20 años, gracias al boom turístico de la Riviera Maya, diversa población de la región ha migrado, colocándose en puestos de trabajo del sector hotelero y restaurantero de la zona.

Respecto al acceso a la vivienda y a la salud, $40.7 \%$ cuenta con agua entubada, 84.6 $\%$ con drenaje, $86.5 \%$ con servicio sanitario y $96.9 \%$ tiene electricidad en el hogar (Inegi, 2021, p. 21). Además, la cabecera municipal cuenta con una clínica de salud.

En materia educativa, el Inegi en su último censo registra que $8.3 \%$ de la población no tiene escolaridad, $62.3 \%$ cuenta con escolaridad básica, $19.2 \%$ ha cursado la educación media superior y solo $10.2 \%$ accede a la educación superior. En la cabecera municipal existen varias escuelas primarias, dos secundarias, un colegio de bachilleres y la UIMQROO. En lo que se refiere a la disponibilidad de TIC, el rezago tecnológico de esta región es evidente: $54.9 \%$ de las viviendas cuenta con televisión paga, $15.2 \%$ tiene una computadora, $71.9 \%$ tiene teléfono celular y finalmente $19.7 \%$ cuenta con internet (Inegi, 2021, p. 21).

En los últimos años se ha incrementado el uso de wifi móvil por medio de los teléfonos inteligentes que acceden a $2 \mathrm{G}$ y $3 \mathrm{G}$, porque la mayoría de los hogares de la localidad no cuentan con conexión a internet debido a la falta de fibra óptica por parte de empresas de telecomunicación; además, la conexión es lenta y se satura continuamente. De hecho, en la zona funcionan principalmente conexiones por satélite y antenas locales que no todos pueden pagar.

Durante la investigación se identificaron los siguientes lugares con conexión a internet: nueve cibercafés, tres restaurantes, el palacio municipal y la UIMQROO. Los celulares en la región se han vuelto más asequibles, por lo que son habituales entre la población. Para conectarse a internet compran datos o fichas de conexión en tienditas de algunas comunidades, aunque la calidad de la red varía. 


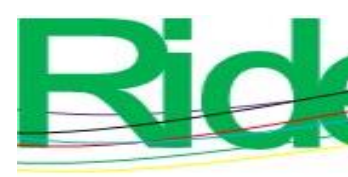

Revista Iberoamericana para la
Investigación y el Desarrollo Educativo
ISSN $2007-7467$

En lo que respecta a la infraestructura en la universidad, en el momento de la investigación se contaba con un salón de 40 computadoras y la conexión a internet tenía una capacidad de 10 megas de banda ancha para toda la comunidad universitaria, lo que se percibía como insuficiente por parte de los profesores, alumnos y egresados: "La conexión es muy muy lenta y eso hace que a veces nos atrasemos cuando queremos hacer investigaciones" (entrevista a alumna de Lengua y Cultura).

Como resultados del estudio, los jóvenes — a pesar de las condiciones localesutilizan las TIC como medio esencial de información, comunicación y ocio. Además, $60 \%$ de los estudiantes y egresados contaban con computadora personal y $95 \%$ tenía teléfono celular. Para la mayoría el celular se ha vuelto un accesorio indispensable: "Mi celular es mi vida, sinceramente, porque ahí está todo: desde fotos, videos; me acompaña a todos lados" (egresado de Desarrollo Municipal en grupo focal).

En cuanto a la conexión a internet, los jóvenes reportaron que dicho servicio solo puede ser costeado por quienes tienen mayores posibilidades económicas: "Muchas veces, aquí en la universidad, esos muchachos a veces no tienen ni para comer en muchos de los casos: ¿cómo van a tener para contratar un servicio de internet?” (entrevista a profesor de ingeniería en TIC).

Aproximadamente $95 \%$ de los jóvenes manifestó que usaba las redes sociales, especialmente Facebook, WhatsApp, Twitter, Instagram y Snapchat. Asimismo, en el ámbito escolar se utilizan para revisar anuncios escolares, descargar y enviar tareas, compartir documentos y comunicarse con la familia o inscribirse en grupos internacionales donde aprenden maya en colectivo. La mayoría de los jóvenes señalaron que habitualmente usaban WhatsApp debido al bajo consumo de datos de dicha aplicación. En cambio, Facebook es señalado como el medio de comunicación preferido durante varias horas al día y a la semana. Las actividades que llevan a cabo con frecuencia media son revisar tareas de la escuela y ver videos y noticias: "Informarme qué pasa a nivel local” (entrevista a alumna de ingeniería en TIC). Un aspecto relevante fue observar que los jóvenes se conectan en diversos lugares:

Antes de tener internet venía aquí al ciber igual a hacer mis tareas o incluso en el parque, porque habían puesto internet igual en el parque para hacer mis trabajos, pues pusieron puntos de acceso. Pero es bastante lento y algunas veces como muchos van se saturaba la red, no podías ingresar y cuando venía acá a la universidad es como para quedarse casi toda la noche o venir de 


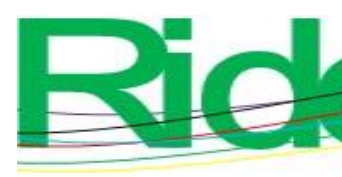

Revista Iberoamericana para la
Investigación y el Desarrollo Educativo ISSN $2007-7467$

madrugada para poder hacer un trabajo (alumna de Ingeniería en TIC en grupo focal).

Algunos alumnos que estudian ingeniería en TIC aprovechan sus conocimientos para usar otras redes de la localidad: “Tengo instalada en mi celular una aplicación que detecta puntos de acceso y te da el código de los que estén vulnerables [risas] y ya me puedo conectar" (alumno de ingeniería en TIC en grupo focal).

Debido a las necesidades que demandan las personas de la localidad, los jóvenes colaboran en la reparación de aparatos digitales de compañeros, amigos y familiares, y los apoyan en la enseñanza de habilidades digitales.

\section{Las trayectorias educativas y la alfabetización digital}

La precariedad tecnológica descrita anteriormente en la educación indígena (INEE, 2019) fue una constante señalada por los jóvenes, así como la escasa infraestructura y las dificultades económicas familiares. De hecho, los estudiantes y egresados indicaron que su primer contacto con una computadora se produjo en secundaria o telesecundaria, cuando tenían entre diez y doce años de edad. Un menor grupo mencionó que utilizó este dispositivo en bachillerato, seguido de la primaria como el nivel educativo que les proporcionó el acceso a las TIC. Incluso, cabe resaltar que un pequeño grupo apenas está aprendiendo las herramientas básicas durante su estancia en la UIMQROO, lo que evidencia un rezago educativo y tecnológico.

El asombro por lo desconocido y la emoción que despertó el uso de las computadoras en la primaria fueron dos aspectos recurrentes que los jóvenes relataron: "Todos nos quedamos asombrados porque ya buscábamos la manera de poder utilizar la computadora en 5. de primaria" (entrevista a alumna de licenciatura en Agroecología).

A su vez, expresaron que en la educación básica (escuelas indígenas) el equipamiento tecnológico era insuficiente. Además, llama la atención que la tecnología era percibida como privilegio en los niveles educativos que cursaron. Por ejemplo, un egresado explicó a que en su primaria las clases de computación eran únicamente para niños que tuvieran influencias con el director, y que como él pertenecía una familia humilde no le fue posible cursar esas clases hasta la secundaria, donde el aprendizaje digital estaba abierto para todos. 


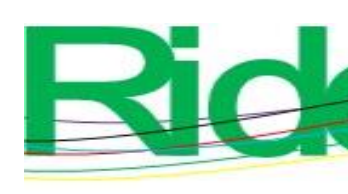

Revista Iberoamericana para la
Investigación y el Desarrollo Educativo
ISSN $2007-7467$

Esta anécdota demuestra que no solo el acceso a las tecnologías digitales está determinado por la infraestructura, sino también por las prácticas locales. Es decir, existen otros factores (en este caso de índole política) que condicionan el uso tecnológico (Andrés, 2014).

Aunado a eso, los jóvenes contaron cómo las clases de computación fueron escasas o de baja calidad, por lo que obtuvieron escasos aprendizajes en el medio escolar, por lo que las visitas a los cibercafés se convirtieron en los espacios informales y autodidactas para desarrollar la alfabetización digital: "Mis compañeros me estuvieron enseñando y viendo tutoriales. Así fue como estuve aprendiendo a utilizarla, yo venía de una educación muy mala" (egresado de licenciatura en Gestión Municipal en grupo focal).

Por otra parte, la ansiedad presupuestaria fue un aspecto recurrente en los relatos, pues los estudiantes se preocupaban al saber que no tendrían cómo pagar si se llegara a “descomponer la computadora" (egresado de licenciatura en Gestión Municipal en grupo focal). Igualmente, se destaca el rol que cumplieron los docentes en el proceso motivacional de los alumnos hacia el aprendizaje.

No obstante, las trayectorias sobre la alfabetización digital se identificaron en su mayoría como tardías, pues se detectaron dificultades en el uso de las herramientas digitales básicas. A partir de los resultados de la encuesta, se percibió que el conocimiento más amplio se hallaba en el uso de la paquetería básica, y no tanto en el empleo, por ejemplo, de hojas de Excel, plataformas digitales académicas y acciones más avanzadas de hardware y software.

Según los docentes consultados, esta realidad depende de la escuela de donde proviene cada estudiante. Para los profesores, se encuentran mejor preparados los alumnos del Centro de Bachillerato Tecnológico Industrial y de Servicios (CBTIS) que los de telebachilleratos, lo que deja entrever la calidad del sistema educativo indígena que prevalece en la localidad: "Yo ya había pasado el bachillerato y no tenía ni idea de cómo utilizar una computadora, ni Word, ni Power Point, tenía como 16 años y no sabía cómo utilizarla” (egresado en Desarrollo Municipal en grupo focal).

Estas carencias se ven reflejadas en el ingreso a la universidad, donde la mayoría llega con deficiencias tecnológicas, razón por la cual la materia de informática fue ubicada en los primeros semestres. 


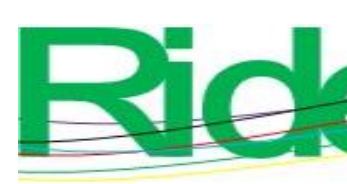

Revista Iberoamericana para la
Investigación y el Desarrollo Educativo
ISSN $2007-7467$

Frente a las diferentes dificultades que se tienen, la activación de las redes de apoyo entre compañeros y la alfabetización digital que se otorga en la universidad intercultural son dos alicientes importantes. Al respecto, un profesor comentó: "También se apoyan de otros chicos, ahí van haciendo interacción y haciendo equipos para hacer trabajos y así también van aprendiendo otras cosas" (entrevista a profesor de lenguas).

A partir de lo anterior, se infiere que el rezago tecnológico tiene una relación estrecha con los factores económicos, educativos y culturales (es decir, pertenecer a un grupo indígena), donde la marginación y la pobreza inciden en el uso y apropiación de las TIC (INEE, 2019).

\section{Los usos significativos de las TIC y las redes sociodigitales}

La apropiación social se refiere a los usos significativos que las personas otorgan a las TIC, identificando beneficios individuales y colectivos. Por ello, es relevante conocer las percepciones que se tienen sobre las tecnologías, los dispositivos y los potenciales usos tomando en cuenta los contextos global y local (Feixa, 2014).

Asimismo, en el conjunto de artefactos y herramientas tecnológicas subyace una interpretación simbólica que — de acuerdo con Pierre Lévy (2007) — corresponde a "una serie de concepciones, representaciones en torno a su invención, producción y utilización: las técnicas son portadoras de proyectos, de esquemas imaginarios, de implicaciones sociales y culturales muy variadas" (p. 7).

Para los jóvenes mayas que participaron en este estudio la tecnología y la conexión a internet se reconocen como una necesidad en el desarrollo del pueblo, donde la brecha digital es una limitante que no permite capitalizar oportunidades: "Ahorita es una comunidad apenas en desarrollo, pues existen infinidad de proyectos para que no esté rezagado el pueblo; la tecnología se necesita" (alumna de ingeniería en TIC en grupo focal).

Los beneficios que se asocian al uso de las TIC son múltiples, como la comunicación entre personas y comunidades, el trabajo remoto, la educación a distancia, los trámites virtuales, el comercio electrónico, el activismo digital, la organización social y política y la revitalización cultural y lingüística: "Últimamente han estado hablando mucho sobre la revitalización de la lengua maya, siento que el Internet es una forma de como nosotros podemos expandir esta información a través de páginas, blogs (...). Son bastante útil” (entrevista a alumna de licenciatura en Lengua y Cultura). 


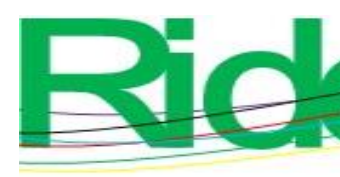

Revista Iberoamericana para la Investigación y el Desarrollo Educativo ISSN $2007-7467$

En cuanto al uso de las redes sociales, se observó el gran impacto que están teniendo tanto en sus formas de consumo cultural como en sus prácticas cotidianas, producto de un entrecruce entre lo global y lo local (Pérez Ruiz, 2019):

Siento que hay una aculturación muy fuerte entre los jóvenes, han cambiado mucho sus hábitos; por ejemplo, están en el celular y están ahí físicamente, pero mentalmente están en otra cosa. En cuestiones de las modas donde las seguimos sin pensar" (egresado de licenciatura en Desarrollo Municipal en grupo focal).

Sobre Facebook, los jóvenes opinan que es una plataforma para comunicarse de manera sencilla con amigos y conocidos, publicar fotos y ver lo que divulgan sus contactos; asimismo, les permite estar informados y descargar libros o videos. Incluso, les ofrece la posibilidad de crear grupos y comunidades sobre temáticas específicas utilizando tanto la lengua maya como el español.

Otros potenciales que observan es el impulso económico mediante proyectos como el ecoturismo, el comercio en la localidad y el potencial que tiene internet y las redes sociales como plataformas de participación ciudadana, donde el activismo político y la creación de movimientos ciudadanos permiten resolver problemas comunitarios:

Sirve para exigir derechos que tenemos... En la colonia que estábamos que nos habíamos quedado sin agua... Estuvimos mandando mensajes por Facebook a la Alcaldía para ver qué estaba pasando... Así estuvimos exigiendo y mandando mensajes a cada rato, como a la semana mandaron pipas de agua (alumna de licenciatura en Gestión Municipal en grupo focal).

\section{Discusión}

Los resultados expuestos demuestran que en muchas localidades se dificulta el acceso a las TIC debido a las pocas posibilidades económicas y a la falta de infraestructura en la región. Esto coincide con las causas principales de desconexión expuestas por el Inegi (2020a) y lo propuesto por Sunkel (2006) respecto a la influencia del poder adquisitivo y el acceso y uso de las nuevas tecnologías.

A su vez, se encontró que los estudiantes que tienen mayor rezago tecnológico y cuentan con menos habilidades digitales proceden de familias pobres de comunidades alejadas a la cabecera municipal. Además, el idioma español se percibe como otra limitante 


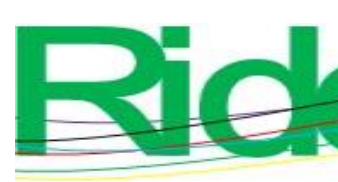

Revista Iberoamericana para la
Investigación y el Desarrollo Educativo
ISSN $2007-7467$

en el aprendizaje de las TIC debido a que las clases no se imparten en la lengua materna de la mayoría de los alumnos de dichas poblaciones. Este hallazgo coincide con el trabajo de Tipa (2019), quien explica que el factor lingüístico está asociado a lo económico en la adquisición de dispositivos y en la alfabetización digital, por lo que se infiere que la brecha digital afecta especialmente a poblaciones pobres e indígenas.

En este contexto de desigualdades, cabe resaltar que la mayoría de los jóvenes de la UIMQROO considera que el uso de la computadora y del internet resulta esencial para la vida académica, lo que se asemeja a lo reportado por Crovi Druetta y López-González (2011) en su investigación, aunque esta haya sido desarrollada con jóvenes urbanos universitarios.

A partir de los relatos de los participantes de la investigación sobre las trayectorias previas, se identificaron un conjunto de factores que inciden en el uso y apropiación tecnológica, donde resalta el rezago educativo y tecnológico en las regiones de origen. En este sentido, se presenta una relación estrecha con los factores económicos, educativos y culturales por pertenecer a un grupo indígena, donde la marginación y la pobreza inciden en el uso y apropiación de las TIC en un continum de exclusión digital (Mariscal, 2020). Estos resultados concuerdan con lo referido por Ortiz y Alarcón (2014) y Tipa (2019) sobre estudiantes indígenas, la desigualdad digital y el rezago tecnológico de la educación indígena del país.

A pesar de las trayectorias discontinuas, los estudiantes y egresados (especialmente de carreras tecnológicas) fungen como intermediarios tecnológicos (Hepp, 2009) en el aprendizaje digital de familiares y amigos, principalmente con sus padres y otros adultos mayores que no cuentan con habilidades digitales y que son el grupo de edad con mayor brecha digital (Inegi, 2020b).

Respecto a los significados que los jóvenes otorgan al uso de las tecnologías digitales, destaca el empleo de las redes sociales en su cotidianidad como parte de una nueva cultura digital (Meneses, 2019), incluso a pesar de que no siempre cuentan con las condiciones ideales de conectividad. A su vez, sobresalen algunos beneficios del uso de las TIC en el entorno local y comunitario. En este panorama, la apropiación de las TIC — según LeónPasquel (2018) — es una realidad donde "la expansión de la conectividad en beneficio de sectores de la población de bajos recursos trae consigo también nuevas destrezas de alfabetismos y habilidades tecnológicas” (p. 48), así como distintas experiencias de usos colectivos de las TIC para el servicio comunitario. 


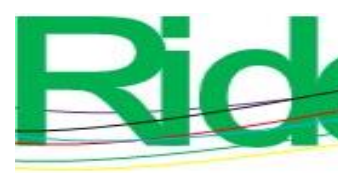

Revista Iberoamericana para la Investigación y el Desarrollo Educativo ISSN $2007-7467$

\section{Conclusiones}

Este estudio arrojó resultados sobre los factores que caracterizan la brecha digital en una zona indígena de México, pero sobre todo muestra un relato acerca del dinamismo que los jóvenes universitarios mayas activan para desafiarla en función de sus posibilidades educativas, económicas y sociales, así como de la motivación por integrarse a lo tecnológico. Cabe mencionar que este grupo corresponde a un sector privilegiado que ha accedido a la educación superior, lo que en el país constituye alrededor de $1 \%$.

Además, la investigación permitió develar la incidencia del contexto local en la apropiación social por medio del conocimiento de los usos y los beneficios de las TIC y redes sociales que otorgan los estudiantes y egresados de la UIMQROO, así como las percepciones sobre las tecnologías digitales y los potenciales en el desarrollo individual y comunitario.

En cuanto a los factores económicos, es clara la relación entre pobreza y desigualdad digital que atraviesa a los jóvenes universitarios mayas, situación estructural e histórica que no ha cambiado en décadas. Sobre los factores educativos, se analizaron las trayectorias escolares en la adquisición de habilidades digitales, las cuales se caracterizan por ser diferentes en cada caso, aunque la falta de infraestructura tecnológica escolar, la alfabetización digital deficiente y las amplias carencias económicas son los denominadores comunes. No obstante, la nueva cultura digital juvenil se afianza con gran fuerza y los jóvenes generan estrategias de conexión y de aprendizaje digital donde las redes de amistad, los docentes y los familiares son un soporte central en el aprendizaje de habilidades digitales.

Cabe mencionar que la adquisición de herramientas digitales tardía no es impedimento para que los alumnos de la UIMQROO estén conectados y para que actúen como intermediarios digitales con sus padres y abuelos o conocidos. Es decir, aunque existan condiciones de rezago educativo y tecnológico, los jóvenes tienden lazos de solidaridad para contribuir al desarrollo comunitario a partir de las TIC, emergiendo como facilitadores digitales.

Finalmente, la universidad se establece como un espacio de socialización digital que los impulsa a la apropiación tecnológica donde aparece la oportunidad de utilizar las TIC para fortalecer a los pueblos indígenas y participar en la cultura digital. 


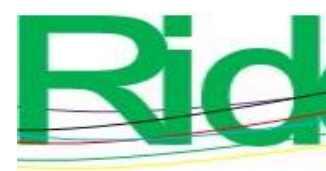

Revista Iberoamericana para la Investigación y el Desarrollo Educativo ISSN $2007-7467$

\section{Futuras líneas de investigación}

El estudio presentado expone la necesidad de seguir profundizando sobre el desarrollo de proyectos de uso de TIC que los estudiantes y egresados de la UIMQROO realizan en sus comunidades para comprender el tipo de habilidades digitales que activan y que se conjuntan con competencias interculturales. A su vez, se vislumbra la pertinencia de comprender el significado que tiene el uso de redes sociodigitales en espacios que van más allá de lo escolar y que inciden en la configuración de identidades juveniles en zonas rurales e indígena.

Por último, se identifican líneas de estudio pendientes para conocer los escenarios y desafíos que surgieron en las propuestas de educación virtual y comunidades de aprendizaje que se pusieron en marcha como parte de la pandemia por covid-19, lo cual demandó a las universidades interculturales de México la generación de estrategias para continuar los cursos a pesar de las precarias condiciones de infraestructura tecnológica y de habilitación digital. 


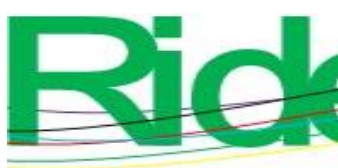

Revista Iberoamericana para la Investigación y el Desarrollo Educativo ISSN $2007-7467$

\section{Referencias}

Andrés, G. (2014). Una aproximación conceptual a la "apropiación social" de TIC. Question/Cuestión, 1(43), 17-31. Recuperado de https://perio.unlp.edu.ar/ojs/index.php/question/article/view/2227

Bardin, L. (1996). Análisis de contenido. Madrid: Akal Ediciones.

Bonilla, M., Cruz, K. y Pérez, R. (2018). Lenguas indígenas en el ciberespacio: retos y desafíos. En Cobo, C., Cortesi, S., Brossi, L., Doccetti, S., Lombana, A., Remolina, N., Winocur, I. R. y Zucchetti, A. (eds.), Jóvenes, transformación digital y formas de inclusión en América Latina (pp.64-74). México: Penguin Random House.

Borrero, M. (2016). Indigenous Peoples and the Information Society: Emerging uses of ICTs. A paper prepared for the First WSIS+10 Review Event, Paris. The United Nations Educational, Scientific and Cultural Organization. Retrieved from http://www.unesco.org/new/fileadmin/MULTIMEDIA/HQ/SC/pdf/UNESCOLINKS_IPs-ICTs.pdf

Boza, C. A., Toscano, C., M. de la O. y Méndez, G. J. M. (2009). El impacto de los proyectos TICS en la organización y los procesos de enseñanza-aprendizaje en los centros educativos. Revista de Investigación Educativa, 27(1), 263-289. Recuperado de https://revistas.um.es/rie/article/view/94401

Cobo, C., Cortesi, S., Brossi, L., Doccetti, S., Lombana, A., Remolina, N., Nelson, N., Winocur, I. R. y Zucchetti, A. (2018). Jóvenes, transformación digital y formas de inclusión en América Latina. México: Penguin Random House.

Cooper, P. (20 de abril de 2020). 140 estadísticas de redes sociales que son importantes para los mercadólogos en 2020. Recuperado de https://blog.hootsuite.com/es/125estadisticas-de-redes-sociales/\#generales

Crovi Druetta, D. y López-González, R. (2011). Tejiendo voces: jóvenes universitarios opinan sobre la apropiación de internet en la vida académica. Revista Mexicana de Ciencias Políticas y Sociales, 56(212), 69-80. Recuperado de http://www.scielo.org.mx/scielo.php?script=sci_arttext\&pid=S0185-

$19182011000200005 \& \operatorname{lng}=$ pt\&tlng=es

Feixa, C. (2014). De la Generación@ a la \#Generación: la juventud en la era digital. Bogotá: Nuevos Emprendimientos Editoriales. 


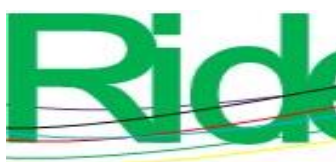

Revista Iberoamericana para la
Investigación y el Desarrollo Educativo
ISSN $2007-7467$

Gallegos, S. (2016). Redes sociales: información, comunicación y sociedad en el siglo XXI (2000-2010) (tesis de doctorado). Facultad de Ciencias de la Documentación. Universidad Complutense de Madrid. Recuperado de https://eprints.ucm.es/44233/1/T39077.pdf

Guber, R. (2011). La etnografía. Método, campo y reflexividad. Buenos Aires: Siglo Veintiuno Editores.

Hepp, P. (2009). Las tecnologías digitales en contextos interculturales. Revista CUHSO, 17(1), 83-89. Doi: https://doi.org/10.7770/cuhso.v17i1.295

Instituto Federal de Telecomunicaciones [IFT] (2018). Cobertura garantizada del servicio móvil en los pueblos indígenas con base en información proporcionada por los concesionarios en el año 2017. Recuperado de http://www.ift.org.mx/sites/default/files/coberturapueblosindigenas_vacc.pdf

Instituto Nacional de Estadística y Geografía [Inegi] (2020a). Encuesta Nacional sobre Disponibilidad y Uso de las Tecnologías de la Información en los Hogares (ENDUTIH) 2019. Comunicado de prensa nro. 216/20. Recuperado de https://www.inegi.org.mx/contenidos/saladeprensa/aproposito/2020/EAP_Internet20 .pdf

Instituto Nacional de Estadística y Geografía [Inegi] (2020b). Encuesta Nacional sobre Disponibilidad y Uso de las Tecnologías de la Información en los Hogares (ENDUTIH) 2019. Tabulados. Recuperado de https://www.inegi.org.mx/programas/dutih/2019/default.html\#Tabulados

Instituto Nacional de Estadística y Geografía [Inegi] (2021). Panorama sociodemográfico de Quintana Roo. Censo de Población y Vivienda 2020. Recuperado de https://www.inegi.org.mx/contenidos/productos/prod_serv/contenidos/espanol/bvine gi/productos/nueva_estruc/702825197964.pdf

Instituto Nacional para la Evaluación de la Educación [INEE] (2019). Panorama educativo de México. Indicadores del Sistema Educativo Nacional 2018. Educación básica y media superior. Recuperado de https://www.inee.edu.mx/wpcontent/uploads/2019/08/P1B117.pdf

León-Pasquel, L. (2018). Entre el mensaje romántico y el etnorock en YouTube: repertorios identitarios en los paisajes virtuales de jóvenes mayas tsotsiles. LiminaR, 16(1), 4055. Doi: https://doi.org/10.29043/liminar.v16i1.563 


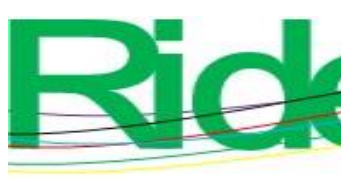

Revista Iberoamericana para la
Investigación y el Desarrollo Educativo
ISSN $2007-7467$

Lévy, P. (2007). Cibercultura. Informe al Consejo de Europa. México, Anthropos y Universidad Autónoma Metropolitana.

Mariscal, J. (2020). A tale of two reforms: Telecommunications reforms in Mexico. Telecommunications Policy, 7(44). Doi: https://doi.org/10.1016/j.telpol.2020.101942

Meneses, J. A. (2019). Estrategias de etnografía multisituada con jóvenes universitari@s indígenas que navegan en Facebook. Etnografías Contemporáneas, 5(9). Recuperado de http://revistasacademicas.unsam.edu.ar/index.php/etnocontemp/article/view/526

Ortiz, V. y Alarcón, E. (2014). Estudiantes, profesores y TIC. La investigación en México. En Ramírez, A. y Casillas, M. A. (coords.), Háblame de TIC: tecnología digital en la educación superior (pp.39-70). México: Editorial Brujas.

Pérez Ruiz, M. L. (2019). Jóvenes indígenas en América Latina: reflexiones para su investigación desde la antropología. Anuario Antropológico, 44(2). Doi: https://doi.org/10.4000/aa.4003

Ramos Mancilla, O. (2020). El agregado digital en las juventudes indígenas: entre desigualdades y representaciones locales. Perspect. ciênc. Informacao, 25(1), 263281.

Recuperado

de

http://www.scielo.br/scielo.php?script=sci_arttext\&pid=S141399362020000100263 $\& \operatorname{lng}=\mathrm{en} \& \mathrm{nrm}=\mathrm{iso}$

Restrepo, E. (2016). Etnografía: alcances, técnicas y ética. Bogotá: Envión Editores.

Robinson, L., Schulz, J., Blank, G., Ragnedda, M., Ono, H., Hogan, B., Mesch, G. S., Cotten, S. R., Kretchmer, S. B., Hale, T. M., Drabowicz, T., Yan, P., Wellman, B., Harper, M.-G., Quan-Haase, A., Dunn, H. S., Casilli, A. A., Tubaro, P., Carvath, R., Chen, W., Wiest, J. B., Dodel, M., Stern, M. J., Ball, C., Huang, K.-T. and Khilnani, A. (2020). Digital inequalities 2.0: Legacy inequalities in the information age. First Monday, 25(7). Doi: https://doi.org/10.5210/fm.v25i7.10842

Santos, M. J. (2003). Introducción. La redefinición cultural de los sistemas tecnológicos. En Bueno, C. y Santos, J. M. (coords.), Nuevas tecnologías y cultura (pp. 7-13). México: Anthropos Editorial y Universidad Iberoamericana.

Secretaría de Comunicaciones y Transportes [SCT] (2019). Programa de cobertura social. Recuperado de https:/www.gob.mx/cms/uploads/attachment/file/500252/2019-1002_PCS_version_web_miercoles_9_octubre.pdf 


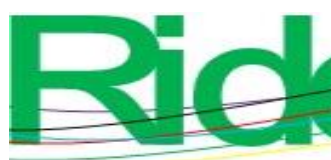

Revista Iberoamericana para la Investigación y el Desarrollo Educativo ISSN $2007-7467$

Soto-Hernández, D., Valencia-López, O. D. y Rentería-Gaeta, R. (2020). Alfabetización y brecha digital entre los pueblos originarios de México, 1990-2015. Efectos socioeconómicos. Trilogía Ciencia Tecnología Sociedad, 12(23). Doi: https://doi.org/10.22430/21457778.1720

Sunkel, G. (2006). Las tecnologías de la información y la comunicación (TIC) en la educación en América Latina: una exploración de indicadores. Santiago de Chile: Naciones Unidas.

Taylor, S. y Bogdan, R. (1987). Introducción a los métodos cualitativos de investigación: la búsqueda de significados. Barcelona: Paidós.

Tipa, J. (2019). Etnicidad, el capital tecnológico y el uso de las tecnologías de información y comunicación. En Pedraza J., Brito, K. y Cortés, E. (coords.), Juventudes latinoamericanas: perspectivas desde la interdisciplinariedad. Seminario Internacional sobre Estudios de Juventud en América Latina (pp.79-100). Centro Avanzado de Estudios en Niñez y Juventud - CINDE. Colectivo CH.

Van Dijk, J. A. (2017). Digital Divide: Impact of Access. In Rössler P., Hoffner C.A. and Zoonen L. (eds.), The International Encyclopedia of Media Effects. University of Twente. Doi: https://doi.org/10.1002/9781118783764.wbieme0043

Velasco, H. y Díaz de Rada, A. (1997). La lógica de la investigación etnográfica. Un modelo de trabajo para etnógrafos de la escuela. Madrid: Trotta.

Vergara, G., (2015). Uso educativo de las tecnologías de información y comunicación en la región indígena Mixe de Oaxaca. El caso del ISIA-Ayuuk (tesis doctoral). Universidad Complutense de Madrid, España.

Winocur, I. R. (2015). La emergencia de esferas público-privadas. En Winocur, I. R. y Sánchez, M. J. A. (eds.), Redes sociodigitales en México (pp.62-80). México: Fondo de Cultura Económica. 